\title{
Extraction of scattering lengths from final-state interactions
}

\author{
A. Gasparyan, ${ }^{1}$ J. Haidenbauer, ${ }^{2}$ and C. Hanhart ${ }^{2}$ \\ ${ }^{1}$ Institute of Theoretical and Experimental Physics, B. Cheremushkinskaya 25, RU-117259 Moscow, Russia \\ ${ }^{2}$ Institut für Kernphysik (Theorie), Forschungszentrum Jülich, D-52425 Jülich, Germany
}

(Received 24 June 2005; published 26 September 2005)

\begin{abstract}
A recently proposed method, based on dispersion theory, that allows the scattering length of a hadronic two-body system to be extracted from corresponding final-state interactions is generalized to the situation where the Coulomb interaction is present. The steps required in a concrete practical application are discussed in detail. In addition a thorough examination of the accuracy of the proposed method is presented and a comparison is made with results achieved with other methods such as the Jost-function approach based on the effective-range approximation. Deficiencies of the latter method are pointed out. The reliability of the dispersion theory method for also extracting the effective range is investigated.
\end{abstract}

DOI: 10.1103/PhysRevC.72.034006

PACS number(s): 25.10.+s, 11.55.Fv, 13.75.Cs, 13.75.Ev

\section{INTRODUCTION}

The scattering length not only provides an important measure for the strength of the interaction in a specific hadronic two-body system [1] but often allows us to draw further more general and thus even more interesting conclusions. For example, in the case of the proton-proton and neutron-neutron systems the corresponding scattering lengths in the ${ }^{1} S_{0}$ partial wave provide a very sensitive test of charge symmetry in the strong interaction [2]. SU(3) symmetry can be tested by comparing the ${ }^{1} S_{0}$ scattering lengths in the neutron-proton and $\Sigma^{+} p$ systems, which should fulfill the relation $a_{n p}=a_{\Sigma^{+} p}$ when SU(3) symmetry holds rigorously [3]. In the chiral limit the $\pi N S$-wave scattering lengths vanish and therefore any deviation from that value is a direct measure of how strongly this symmetry is broken. Here especially the isoscalar component is significant because of its close link to the $\Sigma$ term of the nucleon [4]. On a more phenomenological level the $\eta N$ scattering length is interesting because the magnitude of its real part is directly linked with speculations about the existence of $\eta$-mesonic hadronic bound states such as $\eta^{3} \mathrm{He}[5-11]$ or $\eta^{4} \mathrm{He}$ [12].

Unfortunately, a direct determination of the scattering length is only feasible in a few cases. It can be done with scattering experiments sufficiently close to the reaction threshold so that the effective range expansion can be utilized for extracting the scattering length. But in practice such experiments are only possible for charged and also (quasi) stable particles, as is the case with, for example, $p p, \pi^{+} p$, or $K^{+} p$ scattering. One can also extract the scattering length from a study of the hadronic level shifts of atoms [13] such as $\pi^{-} p, \pi^{-} d$, or also $\bar{p} p$ [14]. For the majority of hadronic two-body systems information about the scattering length is only accessible via an investigation of the final-state interaction of systems that have at least three particles in the final state.

Whereas in the former type of experiments the accuracy of the scattering length is directly connected with the precision of the data, more detailed and often sophisticated considerations are necessary to estimate additional uncertainies that arise when the scattering length is extracted from final-state effects [15]. However, in some cases such an estimation is facilitated by the fact that the reaction mechanism is known. For example, the reaction $n d \rightarrow(n n) p$, one of the prime sources of the $n n$ scattering length, can be analyzed by means of rigorous Faddeev calculations [16-18]. Reactions involving the pion such as $\pi d, \pi^{-} d \rightarrow \gamma n n$ or $\gamma d \rightarrow \pi^{+} n n$, which can be used to extract the $\pi N$ and $n n$ scattering lengths, respectively, can be tackled by chiral perturbation theory in a well-controlled way in the relevant near-threshold regime $[19,20]$.

In a recent publication [21] we argued that also largemomentum transfer reactions such as $p p \rightarrow K^{+} p \Lambda$ [22-24] or $\gamma d \rightarrow K^{+} n \Lambda$ [25-30] are excellent candidates for extracting scattering lengths information. In reactions with large momentum transfer the production process is necessarily of a short-range nature. As a consequence, the results are basically insensitive to details of the production mechanism and therefore reliable and general error estimation can be given. Indeed, in Ref. [21] a formalism based on dispersion theory was presented that relates spectra from large-momentum transfer reactions, such as $p p \rightarrow K^{+} p \Lambda$ or $\gamma d \rightarrow K^{+} n \Lambda$, directly to the scattering length of the interaction of the final-state particles. The theoretical error of the method was estimated to be $0.3 \mathrm{fm}$ or even less, which is comparable to the error quoted in the context of the determination of $a_{n n}$ [31]. This estimate was confirmed by comparing results obtained with the proposed formalism to those of microscopic model calculations for the specific reaction $p p \rightarrow K^{+} \Lambda p$. The arguments but also the formalism of Ref. [21] are, of course, valid for any production or decay process that is of a short-range nature, that is, also for investigation of hadronic two-particle subsystems resulting from the decay of the $J / \Psi$ or $B$ mesons [32,33].

In the present paper we want to investigate further aspects of extracting scattering lengths from final-state interactions that were not addressed in our earlier work. One of those topics is the presence of the Coulomb interaction. In many interesting hadronic two-particle systems both particles carry charges, as in the aforementioned $\Sigma^{+} p$ channel whose scattering length could be extracted from the reaction $p p \rightarrow K^{0} \Sigma^{+} p$. Then the production amplitude acquires additional singularities, owing to the long-range nature of the Coulomb force, and the formalism developed in Ref. [21] is no longer directly 
applicable. We will derive the modifications necessary to adapt the dispersion-relation method to the situation when the Coulomb force is present in the final-state interaction. We also demonstrate in a toy model calculation how one has to proceed in a concrete application to data.

In addition we present a more detailed examination of the accuracy of the method proposed in Ref. [21]. A test based on one specific model calculation, namely for the reaction $p p \rightarrow K^{+} \Lambda p$, has been already performed in that paper. However, here we want to put this investigation on a broader basis by considering final-state interactions of varying strengths, corresponding to a much larger range of values of the scattering length. In addition we take a look at the effective range $r_{e}$ as well, which can be also extracted by the proposed dispersion-integral method. For the effective range a sensible error estimation is not possible, as was already pointed out in Ref. [21], but it is still interesting to examine in concrete applications whether meaningful results could be achieved. Finally, and equally important, we want to compare the present method with the performance of other, approximative treatments of the final-state interaction that are commonly used in the literature to extract information on the scattering length and also the effective range. This concerns in particular the Jost-function approach [34] based on the effective-range approximation and an even simpler approach that relies simply on utilizing the effective-range approximation itself [35]. Thereby, we will show that the latter methods lead to (partly drastic) systematic deviations from the true values and therefore one has to be rather cautious in the interpretation of results achieved with those methods.

The paper is structured in the following way: In the subsequent section we give a short review of the dispersion integral method for extracting the scattering length from final-state interactions. In Sec. III results of an examination of the accuracy of this method are presented. Thereby, we consider various (singlet and triplet) $S$ wave $Y N$ and $N N$ interactions and compare the scattering lengths and effective range extracted with the dispersion-integral method from appropriately generated final-state effects with the ones predicted by the models. We also apply two approximative methods for treating final-state effects, namely the Jostfunction approach based on the effective-range approximation (Jost-ERA) and the effective-range approximation itself, and compare their performance with that of our method. In Sec. IV we generalize the dispersion-integral method to the case where a repulsive Coulomb interaction is present in the final state. Test calculations for a final-state interaction including the Coulomb force are then presented in Sec. V and it is discussed in detail how one has to proceed in a practical application. The paper ends with a short summary.

\section{FORMALISM}

Our method, which goes back to an idea of Geshkenbein $[36,37]$, is based on using the dispersion-relation technique. Consider the production amplitude $A_{S}$ of a $2 \rightarrow 3$ reaction. To be concrete we discuss $p p \rightarrow K^{+} p \Lambda$, or $\gamma d \rightarrow K^{+} n \Lambda$, with the $\Lambda N$ system being in an $L=0$ partial wave and a specific spin state $S\left({ }^{1} S_{0}\right.$ or $\left.{ }^{3} S_{1}\right)$. This amplitude depends on the total energy squared, $s=\left(p_{1}+p_{2}\right)^{2}$, the invariant mass squared of the outgoing $\Lambda N$ system, $m^{2}=\left(p_{N}+p_{\Lambda}\right)^{2}$, and the momentum transfer $t=\left(p_{1}-p_{K^{+}}\right)^{2}$, where $p_{1}, p_{2}, p_{N}, p_{\Lambda}$, and $p_{K^{+}}$are the 4-momenta of the two initial particles, final nucleon, lambda, and kaon, respectively. Then one can write down a dispersion relation for this amplitude with respect to $m^{2}$ at fixed $s$ and $t$ :

$$
\begin{aligned}
A_{S}\left(s, t, m^{2}\right)= & \frac{1}{\pi} \int_{-\infty}^{\tilde{m}^{2}} \frac{D_{S}\left(s, t, m^{\prime 2}\right)}{m^{\prime 2}-m^{2}} d m^{\prime 2} \\
& +\frac{1}{\pi} \int_{m_{0}^{2}}^{\infty} \frac{D_{S}\left(s, t, m^{\prime 2}\right)}{m^{\prime 2}-m^{2}} d m^{\prime 2},
\end{aligned}
$$

where $\tilde{m}^{2}$ is the upper boundary of the left-hand cut, $m_{0}^{2}=$ $\left(m_{N}+m_{\Lambda}\right)^{2}$, and

$$
D_{S}\left(s, t, m^{2}\right)=\frac{1}{2 i}\left[A_{S}\left(s, t, m^{2}+i 0\right)-A_{S}\left(s, t, m^{2}-i 0\right)\right]
$$

is the discontinuity of the amplitude along the cuts. We neglect here the contributions from possible kaon-baryon interactions. In case they are not small, they still can be considered as constant (weakly mass dependent) if one chooses the kinematics such that the excess energy of the reaction is significantly larger than the typical range of the $\Lambda N$ interaction, (cf. the discussion in Ref. [21]). The index $S$ denoting the spin state will be suppressed in the following to simplify the notation.

For a purely elastic $\Lambda N$ system, the discontinuity along the right-hand cut would be given by

$$
D\left(s, t, m^{2}\right)=A\left(s, t, m^{2}\right) e^{-i \delta} \sin \delta,
$$

where $\delta$ is the $\Lambda N\left({ }^{1} S_{0}\right.$ or $\left.{ }^{3} S_{1}\right)$ scattering phase shift. Then the solution of Eq. (1) in the physical region reads (see Refs. [38-40])

$A\left(s, t, m^{2}\right)=\exp \left[\frac{1}{\pi} \int_{m_{0}^{2}}^{\infty} \frac{\delta\left(m^{\prime 2}\right)}{m^{\prime 2}-m^{2}-i 0} d m^{\prime 2}\right] \Phi\left(s, t, m^{2}\right)$,

where $\Phi\left(s, t, m^{2}\right)$ contains only left-hand singularities and therefore is a slowly varying function of $m^{2}$. To ensure the fulfillment of this requirement it is important that the momentum transfer $t$ be large. We assume also that there is no bound state in the $\Lambda N$ system.

Consider now a realistic situation where inelastic channels are present-as is the case with $\Lambda N$ owing to the coupling to the $\Sigma N$ channel, say. Then one can write down a formula similar to Eq. (1), but with the integration performed over a finite range of masses [21]:

$$
A\left(m^{2}\right)=\exp \left[\frac{1}{\pi} \int_{m_{0}^{2}}^{m_{\max }^{2}} \frac{\delta\left(m^{\prime 2}\right)}{m^{\prime 2}-m^{2}-i 0} d m^{\prime 2}\right] \tilde{\Phi}\left(m^{2}\right),
$$

where $\tilde{\Phi}\left(\mathrm{m}^{2}\right)$ is again a slowly varying function of $\mathrm{m}^{2}$ given a sufficiently small phase shift $\delta$ in the vicinity of $m_{\max }$ [21]. The upper limit $m_{\max }$ has to be chosen in such a way that the corresponding relative momentum of the $\Lambda N$ system, $p_{\max }$, is 
of the order of the typical scale of the $\Lambda N$ interaction, that is, of the order of $1 / a$ or $1 / r$. Equation (5) can be solved with respect to $\delta[21]$ :

$$
\begin{aligned}
\frac{\delta\left(m^{2}\right)}{\sqrt{m^{2}-m_{0}^{2}}}= & -\frac{1}{2 \pi} \mathbf{P} \int_{m_{0}^{2}}^{m_{\max }^{2}} \frac{\log \left|A\left(m^{\prime 2}\right) / \tilde{\Phi}\left(m_{\max }^{2}, m^{\prime 2}\right)\right|^{2}}{\sqrt{m^{\prime 2}-m_{0}^{2}}\left(m^{\prime 2}-m^{2}\right)} \\
& \times \sqrt{\frac{m_{\max }^{2}-m^{2}}{m_{\max }^{2}-m^{\prime 2}}} d m^{\prime 2} .
\end{aligned}
$$

If one neglects the mass dependence of $\tilde{\Phi}\left(\mathrm{m}^{2}\right)$ and uses the relation between the partial cross section $\sigma_{S}$ and the amplitude,

$$
\frac{d^{2} \sigma_{S}}{d m^{\prime 2} d t} \propto p^{\prime}\left|A_{S}\left(s, t, m^{\prime 2}\right)\right|^{2},
$$

one obtains the expression for the scattering length in terms of observables,

$$
\begin{aligned}
a_{S}= & \lim _{m^{2} \rightarrow m_{0}^{2}} \frac{1}{2 \pi}\left(\frac{m_{\Lambda}+m_{N}}{\sqrt{m_{\Lambda} m_{N}}}\right) \mathbf{P} \int_{m_{0}^{2}}^{m_{\max }^{2}} d m^{\prime 2} \sqrt{\frac{m_{\max }^{2}-m^{2}}{m_{\max }^{2}-m^{\prime 2}}} \\
& \times \frac{1}{\sqrt{m^{\prime 2}-m_{0}^{2}}\left(m^{\prime 2}-m^{2}\right)} \log \left\{\frac{1}{p^{\prime}}\left(\frac{d^{2} \sigma_{S}}{d m^{\prime 2} d t}\right)\right\}
\end{aligned}
$$

and analogously for the effective range $r_{e}$.

\section{ACCURACY OF THE METHOD AND COMPARISON WITH OTHER APPROACHES}

The most important advantage of the method proposed by us [21] is that a reliable estimate for the uncertainty of the extracted scattering length can be given. There are three sources for the uncertainty: (i) a possible influence of the final-state interaction in the other outgoing channels (for the reaction $p p \rightarrow K^{+} \Lambda p$ considered in Ref. [21] this concerns the $K \Lambda$ and $K N$ systems); (ii) the adopted value for $m_{\max }^{2}$, the upper limit chosen for the dispersion integral in Eq. (7); and (iii) a sensitivity to left-hand cuts of the production operation. A detailed analysis of issues (ii) and (iii), based on general arguments, presented in Ref. [21] suggests that the error in the scattering length should be typically of the order of $0.3 \mathrm{fm}$ or less. The role of issue (i) cannot be quanitified theoretically but has to be investigated by performing experiments and corresponding analyses at different beam momenta [21].

In this section we want to present a thorough examination of the accuracy of the proposed method and, in particular, to corroborate the error estimate, by means of concrete model calculations. A test based on one specific model calculation, namely for the reaction $p p \rightarrow K^{+} \Lambda p$, has already been performed in Ref. [21]. However, here we want to put this investigation on a broader basis by considering final-state interactions of varying strengths, corresponding to a much larger range of values of the scattering length. Furthermore, and equally important, we want to compare the present method with the performance of other, approximative treatments of the final-state interaction that are commonly used in the literature to extract information on the scattering length and the effective range and that have been applied to $p p \rightarrow K^{+} \Lambda p[41,42]$.
One of those approximative treatments follows from the assumption that the phase shifts are given by the first two terms in the effective-range expansion,

$$
p \cot \left(\delta\left(m^{2}\right)\right)=-\frac{1}{a}+\frac{r_{e}}{2} p^{2},
$$

usually called the effective range approximation (ERA), over the whole energy range. Here $p$ is the relative momentum of the final-state particles under consideration in their center-of-mass system, corresponding to the invariant mass $m^{2}$. In this case the relevant integrals (4) can be evaluated in closed form as [34]

$$
A\left(m^{2}\right) \propto \frac{\left(p^{2}+\alpha^{2}\right) r_{e} / 2}{-1 / a+\left(r_{e} / 2\right) p^{2}-i p},
$$

where $\alpha=1 / r_{e}\left(1+\sqrt{1-2 r_{e} / a}\right)$. Because of its simplicity Eq. (9) is often used for the treatment of the final-state interaction (FSI).

A further simplification can be made if one assumes that $a \gg r_{e}$. This situation is practically realized in the ${ }^{1} S_{0}$ partial wave of the $N N$ system. Then the energy dependence of the quantity in Eq. (9) is given by the energy dependence of the elastic amplitude

$$
A\left(m^{2}\right) \propto \frac{1}{-1 / a+\left(r_{e} / 2\right) p^{2}-i p},
$$

as long as $p \ll 1 / r_{e}$. Therefore one expects that, at least for small kinetic energies, $N N$ elastic scattering and meson production in $N N$ collisions with a $N N$ final state exhibit the same energy dependence [34,35], which indeed was experimentally confirmed. This treatment of FSI effects is often referred to as Migdal-Watson (MW) approach [35].

To examine the reliability of the three methods just described we took different $Y N$ models from the literature [43-46] and calculated the production amplitude $A\left(\mathrm{~m}^{2}\right)$ utilizing the meson exchange model from Ref. [47]. This amplitude was then used for extracting the scattering length by means of the dispersion integral Eq. (7) or from the approximative prescriptions given by Eqs. (9) and (10). For comparison we considered also the ${ }^{1} S_{0}$ partial wave of the $n p$ system of the Argonne potential [48]. In this case $A\left(\mathrm{~m}^{2}\right)$ was set equal to the scattering wave function $\Psi(p, r)$ at the origin, more precisely to $\Psi^{-}(p, 0)^{*}$, which corresponds to the assumption that the production operator is pointlike.

Some selective results (for the Nijmegen NSC97 [44] and Jülich 01 [45] $Y N$ models and the Argonne v14 [48] $N N$ potential) are summarized in Table I. The second column lists the correct scattering length evaluated directly from the potential model. One can see that the extraction of the scattering length via the dispersion integral (7) yields results pretty close to the original values for all considered potentials. In fact, in most cases the deviation is significantly smaller than the uncertainty of the method, estimated in Ref. [21] to be $0.3 \mathrm{fm}$. The results of the Jost-ERA approach, Eq. (9), exhibit a systematic offset of the order of $0.3 \mathrm{fm}$. The situation is much worse for the MW approach, Eq. (10), where a similar offset is found though now of the order of $0.6 \mathrm{fm}$. As a consequence, the extracted values differ by $50 \%$ or more from the correct scattering lengths. Only for the ${ }^{1} S_{0} n p$ partial wave does the disagreement remain of the order of $5 \%$. Here the reliability 
TABLE I. $S$-wave scattering lengths $a$ (in fm) for various $Y N$ [44,45] and $N N$ [48] potentials. The results for the original models are compared with those obtained by applying the dispersion-integral method (7) and the approximations of Eq. (9) (Jost-ERA) and Eq. (10) (MW).

\begin{tabular}{lcccc}
\hline \hline Model & Exact result & Dispersion integral & Jost-ERA & MW \\
\hline Jülich 01 singlet & -1.02 & -1.03 & -1.28 & -1.67 \\
Nijmegen 97a singlet & -0.73 & -0.75 & -0.98 & -1.33 \\
Nijmegen 97f singlet & -2.59 & -2.57 & -2.96 & -3.35 \\
Jülich 01 triplet & -1.89 & -1.66 & -2.05 & -2.42 \\
Nijmegen 97a triplet & -2.13 & -1.98 & -2.37 & -2.75 \\
Nijmegen 97f triplet & -1.69 & -1.61 & -2.00 & -2.37 \\
Argonne v14 singlet & -23.71 & -23.54 & -24.56 & -24.79 \\
\hline \hline
\end{tabular}

of the Jost-ERA and MW approaches are comparable. This is in agreement with the aforementioned expectations.

The systematic offset inherent in the Jost-ERA approach as well as in the MW prescription can be best seen in Fig. 1, where we shown the difference between the scattering lengths predicted by various models and the values extracted via the dispersion-integral method (circles), the Jost-ERA method (squares), and the MW prescription (triangles).

Although the Jost-ERA approach might still be a reasonable tool for getting a first rough estimate of the scattering length for a particular two-body interaction one should be rather cautious when using it for more quantitative analyses. In particular, its application in a combined fit to elastic scattering data and invariant mass spectra (e.g., to $\Lambda p$ and $p p \rightarrow K^{+} \Lambda p$ ) is rather problematic and can easily cause misleading results. Because of the offset in the scattering length in applications to final-state effects it is clear that a combined fit cannot converge to a unique ("true") $\Lambda p$ scattering length. Only the elastic data will favor values close to the "true" scattering length, whereas the production data tend to support larger (negative) values. This is obvious from the corresponding Jost-ERA results presented in Table I and also from Fig. 1.

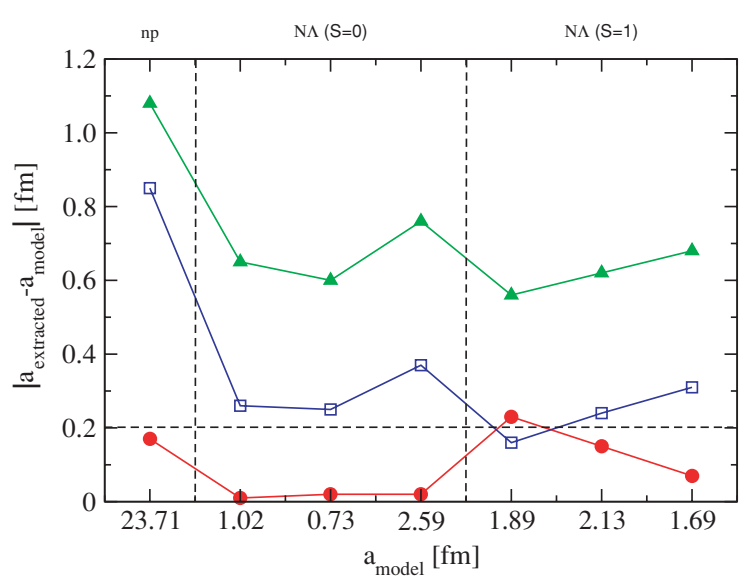

FIG. 1. (Color online) Comparison of different extraction methods for the scattering length $a$. Shown are the differences among results predicted by various $Y N$ and $N N$ models and corresponding values extracted via the dispersion integral method (circles), the Jost-ERA approach (9) (squares), and the MW prescription (10) (triangles). The lines are drawn to guide the eye.
We believe that the analysis of Hinterberger and Sibirtsev presented in Ref. [42] is an instructive exemplification of this dilemma. Employing the Jost-ERA approach to low-energy total $\Lambda p$ cross sections $[49,50]$ and to experimental results for the missing-mass spectrum of the reaction $p p \rightarrow K^{+} X$ [51] separately, they derived (spin-averaged) scattering lengths of $a=-1.81_{-0.21}^{+0.18}$ and $-2.57_{-0.23}^{+0.20} \mathrm{fm}$, respectively. Taking into account the error bars we see that this is roughly the difference we would expect from the offset (of around $0.3 \mathrm{fm}$ ) seen in our test calculations and, therefore, one must consider the results as being practically consistent with each other. But the authors of Ref. [42] attempted to "reconcile" the results even more by introducing a spin dependence in the fitting procedure. Indeed, with the relative magnitude of singlet to triplet contribution in the production reaction as a free parameter (their relative strength in the elastic channel being fixed at 1:3 by the spin weight!) a "fully consistent" description of the combined data could be achieved [42] and apparently the spin-singlet as well as spin-triplet $\Lambda p S$-wave scattering lengths could be determined from spin-averaged observables. Our experience with the Jost-ERA approach reported here, however, strongly suggests that the sensitivity to the spin seen in this analysis is most likely just an artifact of the method applied.

Let us now come to the effective range $r_{e}$. Since the dispersion relations yield only an integral representation for the product $a^{2}\left((2 / 3) a-r_{e}\right)$, but not for the effective range $r_{e}$ alone [21], it follows that the attainable accuracy of $r_{e}$ is always limited roughly by twice the relative error on $a$. Still, it is interesting to see what values one gets for $r_{e}$ from the dispersion integrals. Corresponding results are presented in Table II and in Fig. 2. Evidently, the values extracted via the dispersion integral agree much better with the original results as one might have expected. In fact, in practically all cases the deviation is of the order of only $5 \%$ or even less. This suggests that one could use the dispersion integrals also to extract the effective range $r_{e}$ from the data. But one should keep in mind that, unlike the case of the scattering length, now one cannot rely on a solid and general estimate of the uncertainty. As far as the Jost-ERA approach is concerned it is clear from Table II that it yields rather poor results. In case of the MW prescription (10) it turned out that the fit always prefers an effective range $r_{e}$ equal to zero. This is due to the term proportional to $r_{e}^{2} p^{4}$ in the denominator of the $A\left(\mathrm{~m}^{2}\right)$, which makes the production 
TABLE II. $S$-wave effective ranges $r_{e}$ (in fm) for various $Y N$ [44,45] and $N N$ [48] potentials. The results for the original models are compared with those obtained by applying the dispersion-integral method (7) and the approximation of Eq. (9) (Jost-ERA).

\begin{tabular}{lccc}
\hline \hline Model & Exact result & Dispersion integral & Jost-ERA \\
\hline Jülich 01 singlet & 4.49 & 4.31 & 2.48 \\
Nijmegen 97a singlet & 6.01 & 4.78 & 2.81 \\
Nijmegen 97f singlet & 3.05 & 2.82 & 1.60 \\
Jülich 01 triplet & 2.57 & 2.49 & 1.89 \\
Nijmegen 97a triplet & 2.74 & 2.60 & 1.70 \\
Nijmegen 97f triplet & 3.34 & 2.67 & 1.69 \\
Argonne v14 singlet & 2.78 & 2.91 & 0.43 \\
\hline \hline
\end{tabular}

cross section decrease too fast as compared to the data (or to our calculations with realistic models). Therefore we do not show any results of the MW fit for $r_{e}$.

One should note here that the upper limit in the dispersion integrals was always taken such that $p_{\max }=205 \mathrm{MeV} / c$ (as in Ref. [21]), which corresponds to $\epsilon_{\max } \equiv m_{\max }-m_{0} \approx$ $40 \mathrm{MeV}$ for the $\Lambda N$ and $\Sigma N$ systems and $\epsilon_{\max } \approx 45 \mathrm{MeV}$ for the $N N$ system. In the latter case it is interesting to see what happens if one varies the range of integration, since the energy structure in the $N N$ interaction is much narrower owing to the large $N N$ scattering length. For $\epsilon_{\max }=10$ and $20 \mathrm{MeV}$ as upper limits of the integration one gets the scattering lengths $a=-22.62$ and $-23.17 \mathrm{fm}$, respectively, which are in principle still close to the original value. For the effective range, however, the calculation yields $r_{e}=4.78$ and $3.72 \mathrm{fm}$, respectively. The reason why the agreement for $\epsilon_{\max }>$ $40 \mathrm{MeV}$ is so good is that the $N N^{1} S_{0}$ phase shift becomes sufficiently small at such energies, which implies a small uncertainty according to the error estimation in Ref. [21].

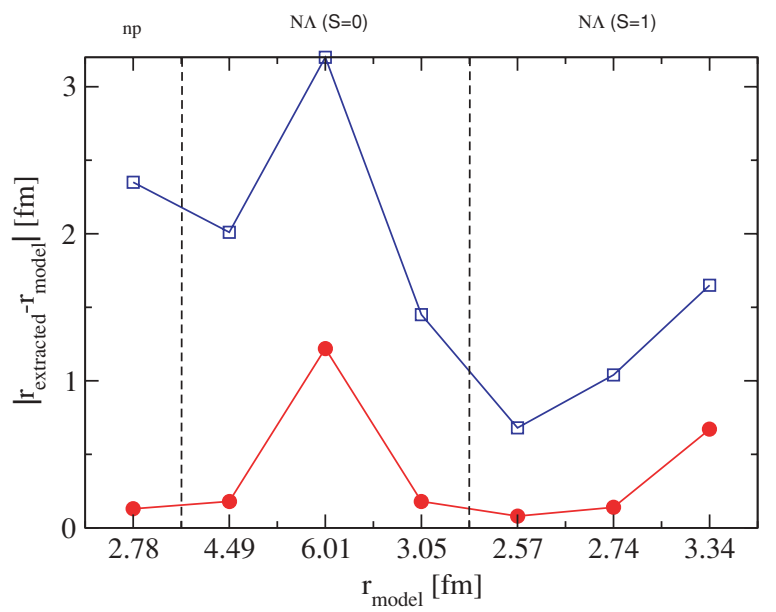

FIG. 2. (Color online) Comparison of different extraction methods for the effective range $r_{e}$. Shown are the differences among results predicted by various $Y N$ and $N N$ models and corresponding values extracted via the dispersion integral method (circles) and the Jost-ERA approach (9) (squares). The lines are drawn to guide the eye.

\section{DISPERSION RELATION IN THE PRESENCE OF COULOMB REPULSION}

In the case when both baryons in the final state carry charges (for example, in the reaction $p p \rightarrow K^{0} p \Sigma^{+}$) there is a Coulomb interaction between them. Then the production amplitude $A\left(\mathrm{~m}^{2}\right)$ acquires additional singularities at $p=0$, owing to the long-range nature of the Coulomb force, and the formalism developed in Sec. II is no longer applicable directly. In this section we describe the modifications necessary to adapt the dispersion-relation method to the situation when the Coulomb force is present in the final-state interaction. We restrict ourselves to the case of a repulsive Coulomb interaction so that no bound states are present.

To elucidate the principle idea we start with the case of elastic (two-body) scattering. Here the problem can be most conveniently dealt with by applying the Gell-MannGoldberger two-potential formalism [52]. Let us assume that the total potential $V=V_{c}+V_{s}$ is given by the sum of a shortranged hadronic potential $V_{s}$ and the Coulomb interaction $V_{c}$. Then the total reaction amplitude $T$ can be written as $T=T_{c}+T_{c s}$, where $T_{c}$ is the Coulomb amplitude and $T_{c s}$ is defined by

$$
T_{c s}=\left(1+T_{c} G_{0}\right) t_{c s}\left(1+G_{0} T_{c}\right),
$$

where $t_{c s}$ fulfills a Lippmann-Schwinger equation,

$$
t_{c s}=V_{s}+V_{s} G_{c} t_{c s},
$$

with the short-range potential $V_{s}$ as driving term. To obtain the physical on-shell amplitudes one needs to project the corresponding $T$ operators on the so-called Coulombian asymptotic states $\left|p_{\infty} \pm\right\rangle$, which are related to the Coulomb scattering states (with fixed angular momentum-in our case $l=0$ ) $|p \pm\rangle_{c}$ via $|p \pm\rangle_{c}=\left|p_{\infty} \pm\right\rangle+G_{0}^{ \pm} T_{c}^{ \pm}\left|p_{\infty} \pm\right\rangle$ [53]. Here $p$ denotes the center-of-mass momentum in the baryon-baryon system. In this way one obtains in particular

$$
{ }_{c}\left\langle p-\left|t_{c s}\right| p+\right\rangle_{c}=-\frac{1}{\pi \mu} f_{c s}(p),
$$

where $f_{c s}$ is the so-called Coulomb-modified nuclear scattering amplitude and $\mu$ is the reduced mass. Its relation to the 
phase shift $\delta_{c s}$ is the following:

$$
f_{c s}=\frac{e^{2 i \delta_{c}}\left(e^{2 i \delta_{c s}}-1\right)}{2 i p},
$$

with $\delta_{c}$ denoting the pure Coulomb $S$-wave phase shift given by $\delta_{c}=\arg (\Gamma(1+i \eta))$ with $\eta=\mu e^{2} / p$.

It has been shown in Ref. [54] under rather general assumptions that the modified amplitude $\tilde{f}(p)=e^{-2 i \delta_{c}} f_{c s}(p) / C^{2}(p)$ is free of Coulomb singularities on the physical sheet and possesses only singularities caused by dynamical cuts (see also Refs. [55-58]). In addition below the inelastic cuts and above the two-baryon threshold the modified unitarity relation reads

$$
\tilde{f}(s+i 0)-\tilde{f}(s-i 0)=2 i p \tilde{f}(p) \tilde{f}^{*}(p) C^{2}(p),
$$

with $C^{2}(p)=2 \pi \eta /\left(e^{2 \pi \eta}-1\right)$ being the Coulomb penetration factor.

Furthermore, an effective-range function, modified for the presence of the Coulomb interaction, can be defined as well. It is given by

$$
S(p) \equiv p C^{2}(p) \cot \delta_{c s}(p)+Q(p)=-1 / a_{c s}+r_{e} p^{2} / 2+\ldots,
$$

where $Q(p) \equiv \mu e^{2}[\psi(i \eta)+\psi(-i \eta)-2 \ln \eta]$ and $\psi(z)=$ $\Gamma^{\prime}(z) / \Gamma(z)$.

Returning now to the production reaction we can analogously show that the modified production amplitude

$$
\tilde{A}\left(m^{2}\right)=e^{-i \delta_{c}} A\left(m^{2}\right) / C(p)
$$

is also free of the aforementioned singularities [54]. Therefore a dispersion relation similar to Eq. (1) can be written as

$$
\tilde{A}\left(m^{2}\right)=\frac{1}{\pi} \int_{-\infty}^{\tilde{m}^{2}} \frac{\tilde{D}\left(m^{\prime 2}\right)}{m^{\prime 2}-m^{2}} d m^{\prime 2}+\frac{1}{\pi} \int_{m_{0}^{2}}^{\infty} \frac{\tilde{D}\left(m^{\prime 2}\right)}{m^{\prime 2}-m^{2}} d m^{\prime 2} .
$$

Unitarity implies that the discontinuity for the elastic cut is

$$
\tilde{D}\left(m^{2}\right)=\tilde{A}\left(m^{2}\right) e^{-i \delta_{c s}} \sin \delta_{c s} .
$$

The solution to Eq. (18), found in complete analogy to the case without the presence of the Coulomb interaction, is

$$
\tilde{A}\left(m^{2}\right)=\exp \left[\frac{1}{\pi} \int_{m_{0}^{2}}^{m_{\max }^{2}} \frac{\delta_{c s}\left(m^{\prime 2}\right)}{m^{\prime 2}-m^{2}-i 0} d m^{\prime 2}\right] \tilde{\Psi}\left(m^{2}\right),
$$

where $\tilde{\Psi}\left(m^{2}\right)$ is some function slowly varying with $m^{2}$. If one neglects the weak $m^{2}$ dependence present in $\tilde{\Psi}\left(m^{2}\right)$ the expression for the phase shift $\delta_{c s}$ in terms of the differential cross section becomes

$$
\begin{aligned}
\frac{\delta_{c s}\left(m^{2}\right)}{\sqrt{m^{2}-m_{0}^{2}}}= & -\frac{1}{2 \pi} \mathbf{P} \int_{m_{0}^{2}}^{m_{\max }^{2}} \frac{\log \left[\frac{1}{p^{\prime} C^{2}\left(p^{\prime}\right)} \frac{d^{2} \sigma}{d m^{\prime 2} d t}\right]}{\sqrt{m^{\prime 2}-m_{0}^{2}}\left(m^{\prime 2}-m^{2}\right)} \\
& \times \sqrt{\frac{m_{\max }^{2}-m^{2}}{m_{\max }^{2}-m^{\prime 2}}} d m^{\prime 2} .
\end{aligned}
$$

Using the effective-range expansion (16) one can then extract the scattering length $a_{c s}$ from this dispersion integral.

\section{TEST OF THE METHOD FOR THE COULOMB CASE}

One of the obvious reactions for applying the formalism including the Coulomb force is $p p \rightarrow K^{0} \Sigma^{+} p$, where one could determine the $\Sigma N$ scattering length for the isospin $3 / 2$ state. Note that the $\Sigma^{+} p$ channel does not couple to the $\Lambda N$ system and is therefore free of inelastic cuts (which start already on the left-hand side) as required for the applicability of the disperson-integral method. For this reaction one could perform a model calculation analogous to the one for $p p \rightarrow K^{+} \Lambda p$ [47], that we used for testing the dispersion-integral method in the absence of the Coulomb interaction [21]. However, implementing Coulomb effects into our momentum-space code is technically complicated and requires some approximations [59]. Thus, for the present test calculation we adopt a different strategy. First, instead of the momentum-space $Y N$ models of Refs. $[43,45,46]$ we take the $r$-space Argonne $(N N)$ potential, however, with parameters modified in such a way that the effective-range parameters are similar to those predicted by realistic $Y N$ potentials [43-46] for the $\Sigma N I=3 / 2{ }^{1} S_{0}$ partial wave. In particular, we prepared two models with Coulomb-modified scattering lengths of $a_{c s}=-3.24 \mathrm{fm}$ (model 1) and $a_{c s}=-1.86 \mathrm{fm}$ (model 2), respectively. The corresponding scattering lengths without the Coulomb interaction are -4.11 and $-2.01 \mathrm{fm}$, respectively. For the transition amplitude we use the scattering wave function calculated from those potential models and evaluated at the origin. This corresponds to the assumption that the production operator is pointlike, which is reasonable as long as we are interested only in the mass dependence of the production amplitude. The corrections stemming from a possible mass dependence of the production operator were discussed in Ref. [21].

The results of applying Eq. (7) with $m_{\max }-m_{0}=$ $40 \mathrm{MeV}\left(p_{\max }=205 \mathrm{MeV} / \mathrm{c}\right)$ are shown in Fig. 3, where we plot the function $1 / S(p)$, which should coincide with

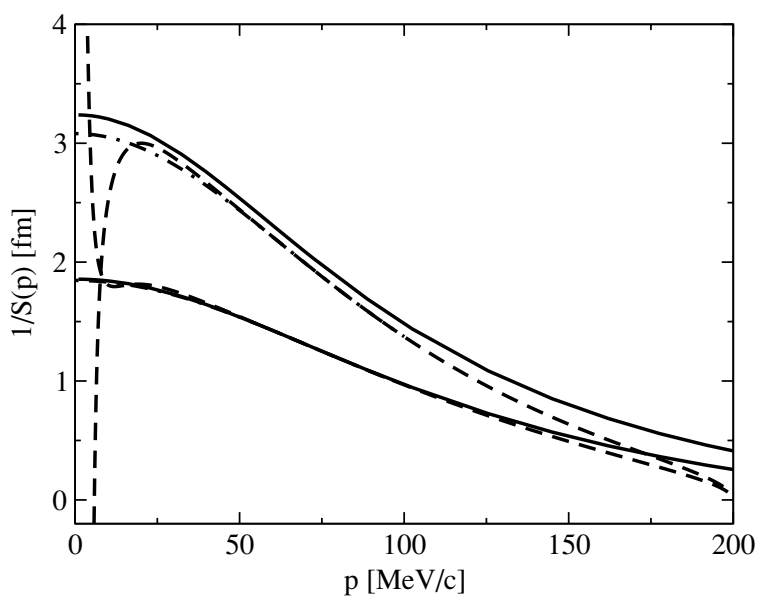

FIG. 3. The inverse of the effective range function $S(p)$ for model 1 (upper curves) and model 2 (lower curves). The solid lines denote the phase shifts predicted by the corresponding model, whereas the dashed lines correspond to the phase shift extracted via Eq. (21). The dash-dotted lines show the result of the smooth extrapolation of the dashed lines, as explained in the text. 
the scattering length $-a_{c s}$ at $p=0$. Obviously, there is a strongly nonanalytic behavior of the extracted inverse effective-range function when approaching the threshold; this behavior, however, can be easily understood. It is clear from Eq. (16) that the threshold behavior of the Coulomb-modified phase shift is $\delta_{c s} \approx-a_{c s} p C^{2}(p)$; that is, $\delta_{c s}$ goes to zero very rapidly. To obtain such a behavior on the left-hand side of Eq. (21) one needs to have a very precise cancellation in the integral on the right-hand side of Eq. (21), which is, of course, impossible if one truncates the integral at a finite momentum. But still one can expect Eq. (21) to work for momenta not too close to the threshold, namely above the typical Coulomb scale of $2 \pi \alpha / \mu \approx 25 \mathrm{MeV} / c$, where the factors $C^{2}(p)$ and $Q(p)$ that appear in the effective-range function [Eq. (16)] become smoother. This is indeed the case, as can be seen from Fig. 3. Thus, a natural and practical step here would be to extrapolate the extracted $S(p)$ to the threshold from above. Using a fourthorder polynomial of the type $-1 / a_{c s}+r_{e} p^{2} / 2-\operatorname{Pr}_{e}^{3} p^{4}$ and fixing the coefficients in the region $50-100 \mathrm{MeV} / c$ (i.e., well above the Coulomb structure), one can then extrapolate $S(p)$ to the threshold (cf. the dash-dotted lines in Fig. 3). In this case one gets a satisfactory agreement between the true and extracted scattering lengths. In fact, the deviations are not worse than in the case when we consider the same potentials without a Coulomb interaction and they are also within the theoretical error of $0.3 \mathrm{fm}$ estimated in Ref. [21]. The extracted values are $a_{c s}=-3.10$ and $-1.86 \mathrm{fm}$ for models 1 and 2, respectively, with a Coulomb interaction and $a_{s}=-4.05$ and $-2.06 \mathrm{fm}$ when the Coulomb interaction is switched off. We also checked that the sensitivity of the result to the region of interpolation of the effecitve-range function $S(p)$ is rather low. For instance, if one shifts the lower bound of this region to $70 \mathrm{MeV} / \mathrm{c}$ the corresponding change in the scattering length will be less then $0.05 \mathrm{fm}$.

If one wishes to consider a more realistic situation, one needs to deal with mass distributions with finite statistical errors, finite mass resolution, and finite binning. To examine this situation we have generated two data sets, corresponding to models 1 and 2 as shown in Fig. 4. We have chosen the binning as well as the mass resolution to be equal to $2 \mathrm{MeV}$ (the same as in the experiment [51] that was analyzed in Ref. [21]) and have selected statistics to be rather high to minimize the influence of the statistical error bars on the results. The excess energy was set to $40 \mathrm{MeV}$ to simplify the simulation. In a realistic situation larger values are preferable to minimize the influence of the meson-baryon interactions (cf. see the corresponding remarks in Ref. [21]). In our test calculation such meson-baryon interactions are neglected anyway.

We start here with the procedure suggested in appendix A of Ref. [21], namely by fitting the cross section with an exponential parametrization of the type

$$
\frac{d^{2} \sigma}{d m^{2} d t}=C^{2}(p) \times \exp \left[C_{0}+\frac{C_{1}^{2}}{\left(m^{2}-C_{2}^{2}\right)}\right] \times \text { phase space. }
$$

This formula fits the generated cross section with the $\chi^{2}$ per degree of freedom of $\chi_{\text {dof }}^{2} \sim 1$ (cf. Fig. 4). A new problem that arises here is that the production amplitude contains a very

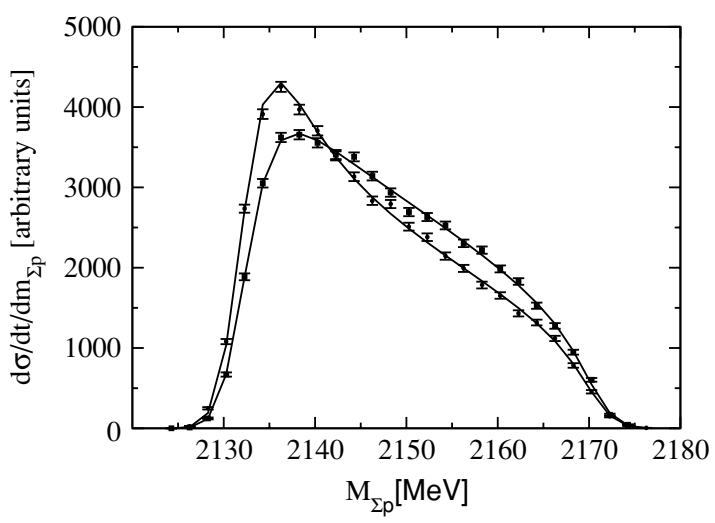

FIG. 4. Pseudo data for the differential $\Sigma^{+} p$ cross section generated from model 1 (circles) and model 2 (squares) as a function of the $\Sigma p$ invariant mass $M_{\Sigma p}$ with corresponding fit by the exponential parametrization, Eq. (22).

narrow structure (of the size $2 \pi \alpha / \mu$ ) close to threshold, as can be seen in Fig. 5 (solid lines). Clearly, this structure cannot be reproduced after the fitting procedure as it gets smeared out by the mass resolution and binning (their size being much larger than the scale of the structure). The amplitudes coming from the fit are depicted in Fig. 5 by dash-dotted lines. However, the fit can be improved if one notes that the structure comes mostly from the part of the dispersion integral (20) containing the leading term in the $\delta_{c s}$ expansion near the threshold, namely (in the nonrelativistic case)

$\exp \left[\frac{1}{\pi} \int_{0}^{\infty} \frac{-a_{c s} C^{2}\left(p^{\prime}\right)}{p^{\prime 2}-p^{2}-i 0}\left(\frac{p^{2}}{p^{\prime 2}}\right) d p^{\prime 2}\right]=\exp \left[-a_{c s} Q(p)\right]$,

where we made a subtraction at $p=0$ to render the integral convergent. This does not change the energy dependence of the

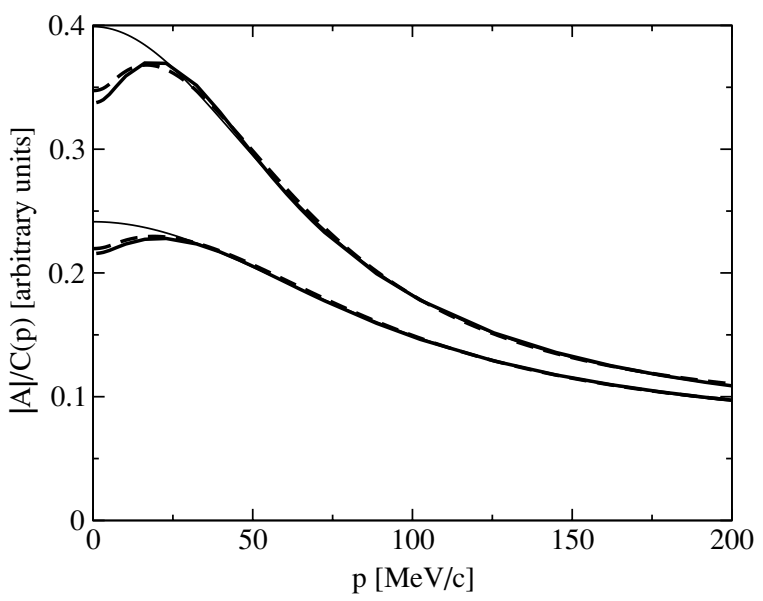

FIG. 5. The production amplitude $A\left(\mathrm{~m}^{2}\right)$ divided by the factor $C(p)$ for model 1 (upper curves) and model 2 (lower curves). The solid lines are the amplitudes as calculated from the models. The dash-dotted lines correspond to the fitted amplitudes. The dashed lines denote the fitted amplitudes improved by the iterative procedure as discussed in the text. 


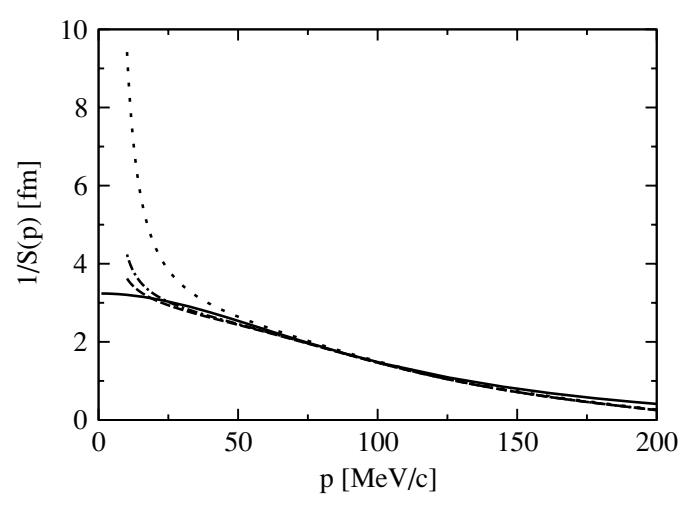

FIG. 6. The inverse of the effective range function $S(p)$ calculated by means of the iterative procedure as discussed in the text. The dotted line corresponds to the zeroth iteration, the dashed line corresponds to the first iteration, and the dash-dotted line corresponds to the second iteration. The solid curve denotes the exact result. Shown are results for model 1.

resulting exponent. Indeed, the structure disappears after dividing the production amplitude by the factor $\exp \left[-a_{c s} Q(p)\right]$.

Obviously the scattering length is unknown before its extraction! But one can resort to an iterative procedure by first including the extraction of the unimproved scattering length, then putting it into the fit function,

$$
\begin{aligned}
\frac{d^{2} \sigma}{d m^{2} d t}=C^{2}(p) \times \exp [ & C_{0}+\frac{C_{1}^{2}}{\left(m^{2}-C_{2}^{2}\right)} \\
& \left.-2 a_{c s} Q(p)\right] \times \text { phase space, }
\end{aligned}
$$

and by repeating this step until the procedure converges. Fortunately, convergence is attained after only three or four iterations, and the resulting amplitudes are shown in Fig. 5 by the dashed lines. The improvement of the fit is quite obvious. Finally, we applied the combination of the extrapolation and iteration procedures to obtain the scattering lengths from the pseudo data. Since the data have a statistical uncertainty we generated a sample of 1000 mass distributions and looked at the average value of the scattering lengths. They turned out to be $-2.89 \pm 0.06 \mathrm{fm}$ for model 1 and $-1.82 \pm 0.05$ for model 2 . Note that the deviation from the correct values is now a bit larger but still reasonable $(0.35 \mathrm{fm}$ in the worst case). Figure 6 shows how the extracted inverse effective-range function approaches the correct one given by the model by the example of model 1.

\section{SUMMARY}

In a recent publication [21] we have presented a formalism based on dispersion relations that allows one to relate spectra from large-momentum transfer reactions, such as $p p \rightarrow$ $K^{+} p \Lambda$ or $\gamma d \rightarrow K^{+} n \Lambda$, directly to the scattering length of the interaction of the final-state particles. An estimation of the systematic uncertainties of that method, relying on general arguments, led to the conclusion that the theoretical error in the extracted scattering length should be less than $0.3 \mathrm{fm}$. This finding was corroborated in an application of the method to results of a microscopic model calculation for $p p \rightarrow K^{+} p \Lambda$.

In the present paper this dispersion theoretical method was generalized to the case where a repulsive Coulomb force is present in the final-state interaction. As an example let us mention the reaction $p p \rightarrow K^{0} p \Sigma^{+}$, which could be used to extract the $p \Sigma^{+}$scattering length. Though the generalization of the formalism itself is straightforward it turned out that there are some additional features owing to the Coulomb interaction that need to be taken into account in concrete applications of the method to data. These practical aspects were thoroughly discussed and it was shown how to circumvent the difficulties. In a test calculation utilizing potential models with effective-range parameters similar to those of realistic $Y N$ interactions the extracted values for the scattering lengths were found to agree within $0.3 \mathrm{fm}$ with those predicted by the models. Thus, the accuracy of the dispersion theoretical method for extracting the scattering lengths from final-state interactions including the Coulomb force is comparable to the case where no Coulomb interaction is present.

We also presented a more detailed examination of the accuracy of the dispersion-integral method than in Ref. [21]. In particular we considered final-state interactions of varying strengths, corresponding to a much larger range of values of the scattering length. These investigations confirmed the reliability of the general error estimate provided in Ref. [21]. Indeed, in most of the considered cases the deviation of the extracted scattering length from the true value was significantly smaller than the uncertainty of $0.3 \mathrm{fm}$ derived in that paper. In addition, we studied the effective range $r_{e}$, which can also be extracted by the proposed dispersion-integral method. For most of the interaction models considered the extracted values of $r_{e}$ agreed remarkably well with the true results. Thus, it might be sensible to use the proposed method to extract the effective range from data - though one should always keep in mind that for this quantity a generally valid error estimation is not possible [21].

Finally, we compared the present method with the performance of other, approximative treatments of the final-state interaction that are commonly used in the literature to extract information on the scattering length and also the effectiverange. of particular we tested the Jost-function approach based on the effective-range approximation (Jost-ERA) and an even simpler approach that relies simply on utilizing the effectiverange approximation itself. Thereby, we showed that the latter methods lead to systematic deviations from the true values of the scattering lengths of the order of $0.3 \mathrm{fm}$ (Jost-ERA) and even $0.7 \mathrm{fm}$ (direct effective-range approximation). This suggests that one should be rather cautious in the interpretation of results achieved with those methods.

\section{ACKNOWLEDGMENTS}

A.G. would like to acknowledge finanical support by Grant No. 436 RUS 17/75/04 of the Deutsche Forschungsgemeinschaft. Furthermore, he thanks the Institut für Kernphysik at the Forschungszentrum Jülich for its hospitality during the period when the present work was carried out. 
[1] See, e.g., C. J. Joachain, Quantum Collision Theory (NorthHolland, Amsterdam, 1975).

[2] G. A. Miller, B. M. K. Nefkens, and I. Slaus, Phys. Rep. 194, 1 (1990).

[3] C. B. Dover and H. Feshbach, Ann. Phys. (NY) 198, 321 (1990).

[4] J. Gasser, H. Leutwyler, and M. E. Sainio, Phys. Lett. B253, 252 (1991).

[5] S. Wycech, A. M. Green, and J. A. Niskanen, Phys. Rev. C 52, 544 (1995).

[6] S. A. Rakityansky, S. A. Sofianos, W. Sandhas, and V. B. Belyaev, Phys. Lett. B359, 33 (1995).

[7] V. B. Belyaev, S. A. Rakityansky, S. A. Sofianos, M. Braun, and W. Sandhas, Few-Body. Syst. Suppl. 8, 309 (1995).

[8] S. A. Rakityansky, S. A. Sofianos, M. Braun, V. B. Belyaev, and W. Sandhas, Phys. Rev. C 53, 2043(R) (1996).

[9] A. Fix and H. Arenhövel, Phys. Rev. C 66, 024002 (2002).

[10] A. Sibirtsev, J. Haidenbauer, J. A. Niskanen, and U.-G. Meißner, Phys. Rev. C 70, 047001 (2004).

[11] J. A. Niskanen, A. Sibirtsev, J. Haidenbauer, and C. Hanhart, Int. J. Mod. Phys. A 20, 634 (2005).

[12] Q. Haider and L. C. Liu, Phys. Rev. C 66, 045208 (2002).

[13] V. Antonelli, A. Gall, J. Gasser, and A. Rusetsky, Ann. Phys. (NY) 286, 108 (2001).

[14] D. Gotta, Prog. Part. Nucl. Phys. 52, 133 (2004).

[15] W. R. Gibbs, S. A. Coon, H. K. Han, and B. F. Gibson, Phys. Rev. C 61, 064003 (2000).

[16] D. E. González Trotter et al., Phys. Rev. Lett. 83, 3788 (1999).

[17] V. Huhn et al., Phys. Rev. C 63, 014003 (2000).

[18] J. Deng, A. Siepe, and W. von Witsch, Phys. Rev. C 66, 047001 (2002).

[19] S. R. Beane, V. Bernard, E. Eṕelbaum, U. G. Meissner, and D. R. Phillips, Nucl. Phys. A720, 399 (2003); A. Gardestig and D. R. Phillips, arXiv:nucl-th/0501049.

[20] V. Lensky, V. Baru, J. Haidenbauer, C. Hanhart, A. E. Kudryavtsev, and U. G. Meissner, arXiv:nucl-th/0505039.

[21] A. Gasparyan, J. Haidenbauer, C. Hanhart, and J. Speth, Phys. Rev. C 69, 034006 (2004).

[22] J. T. Balewski et al., Phys. Lett. B420, 211 (1998); S. Sewerin et al., Phys. Rev. Lett. 83, 682 (1999).

[23] R. Bilger et al., Phys. Lett. B420, 217 (1998).

[24] J. T. Balewski et al., Eur. Phys. J. A 2, 99 (1998).

[25] F. M. Renard and Y. Renard, Nucl. Phys. B1, 389 (1967).

[26] B. O. Kerbikov, B. L. G. Bakker, and R. Daling, Nucl. Phys. A480, 585 (1988); B. O. Kerbikov, Phys. At. Nucl. 64, 1835 (2001).

[27] B. Mecking et al., CEBAF proposal PR-89-045, 1989.
[28] R. A. Adelseck and L. E. Wright, Phys. Rev. C 39, 580 (1989).

[29] X. Li and L. E. Wright, J. Phys. G 17, 1127 (1991).

[30] H. Yamamura, K. Miyagawa, T. Mart, C. Bennhold, W. Glöckle, and H. Haberzettl, Phys. Rev. C 61, 014001 (1999).

[31] C. R. Howell et al., Phys. Lett. B444, 252 (1998).

[32] See, e.g., F. A. Harris, Int. J. Mod. Phys. A 20, 445 (2005), and references therein.

[33] See, e.g., J. Schumann, hep-ex/0505098, and references therein.

[34] M. Goldberger and K. M. Watson, Collision Theory (Wiley, New York, 1964).

[35] K. Watson, Phys. Rev. 88, 1163 (1952); A. B. Migdal, Sov. Phys. JETP 1, 2 (1955) .

[36] B. V. Geshkenbein, Yad. Fiz. 9, 1232 (1969) [Sov. J. Nucl. Phys. 9, 720 (1969)].

[37] B. V. Geshkenbein, Phys. Rev. D 61, 033009 (2000).

[38] N. I. Muskhelishvili, Singular Integral Equations (Noordhof, Groningen, 1953).

[39] R. Omnes, Nuovo Cimento 8, 316 (1958).

[40] W. R. Frazer and J. R. Fulco, Phys. Rev. Lett. 2, 365 (1959).

[41] J. T. Balewski et al., Phys. Lett. B420, 211 (1998); S. Sewerin et al., Phys. Rev. Lett. 83, 682 (1999).

[42] F. Hinterberger and A. Sibirtsev, Eur. Phys. J. A 21, 313 (2004).

[43] B. Holzenkamp, K. Holinde, and J. Speth, Nucl. Phys. A500, 485 (1989).

[44] T. A. Rijken, V. G. J. Stoks, and Y. Yamamoto, Phys. Rev. C 59, 21 (1999).

[45] J. Haidenbauer, W. Melnitchouk, and J. Speth, AIP Conf. Proc. 603, 421 (2001), [arXiv:nucl-th/0108062].

[46] J. Haidenbauer and U.-G. Meißner, nucl-th/0506019.

[47] A. Gasparian, J. Haidenbauer, C. Hanhart, L. Kondratyuk, and J. Speth, Phys. Lett. B480, 273 (2000).

[48] R. B. Wiringa, R. A. Smith, and T. L. Ainsworth, Phys. Rev. C 29, 1207 (1984).

[49] G. Alexander et al., Phys. Rev. 173, 1452 (1968).

[50] B. Sechi-Zorn et al., Phys. Rev. 175, 1735 (1968).

[51] R. Siebert et al., Nucl. Phys. A567, 819 (1994).

[52] M. Gell-Mann and M. L. Goldberger, Phys. Rev. 91, 398 (1953).

[53] H. van Haeringen, J. Math. Phys. 17, 995 (1976).

[54] J. Hamilton, I. Oeverboe, and B. Tromborg, Nucl. Phys. B60, 443 (1973).

[55] H. Cornille and A. Martine, Nuovo Cimento 26, 298 (1962).

[56] H. van Haeringen, J. Math. Phys. 18, 927 (1977).

[57] L. Heller and M. Rich, Phys. Rev. 144, 1324 (1966).

[58] A. Scotti and D. Y. Wong, Phys. Rev. 138, B145 (1965).

[59] C. Hanhart, J. Haidenbauer, A. Reuber, C. Schütz, and J. Speth, Phys. Lett. B358, 21 (1995). 